\title{
Latex agglutination for rapid detection of Pseudomonas pseudomallei antigen in urine of patients with melioidosis
}

M D Smith, V Wuthiekanun, A L Walsh, N Teerawattanasook, V Desakorn, Y Suputtamongkol, T L Pitt, N J White
Faculty of Tropical Medicine, Mahidol University, $420 / 6$ Rajvithi Road, Bangkok 10400, Thailand

M D Smith

V Wuthiekanun

A L Walsh

V Desakorn

Y Suputtamongkol

N J White

Department of Microbiology, Sappasitprasong Hospital, Ubon Ratchatani, Thailand $\mathrm{N}$ Teerawattanasook

Nuffield Department of Clinical Medicine, John Radcliffe Hospital, Oxford University, Oxford M D Smith A L Walsh N J White

Laboratory of Hospital Infection, Central Public Health Laboratory, Colindale Avenue, London T L Pitt

Correspondence to: Dr M D Smith.

Accepted for publication 4 August 1994

\begin{abstract}
A latex agglutination test for the detection of Pseudomonas pseudomallei antigen in urine was evaluated for the rapid diagnosis of melioidosis. With unconcentrated urine, antigen was detected in only $18 \%$ of patients with melioidosis overall. However, when urine was concentrated 100fold, antigen was detected in $47 \%$ overall and in $67 \%$ of patients with septicaemia or disseminated infection, in whom a rapid diagnosis is most important. The specificity of the test was $100 \%$. These results compared favourably with an enzyme immunoassay. This latex agglutination test is a simple, rapid and highly specific method of diagnosing meliodosis, and will be particularly useful in areas with limited laboratory facilities.

(f Clin Pathol 1995;48:174-176)
\end{abstract}

Keywords: Pseudomonas pseudomallei, antigen, latex agglutination.

Melioidosis is a potentially fatal infection caused by the Gram negative bacillus Pseudomonas pseudomallei, an environmental saprophyte found in moist soil and water of many tropical countries. Melioidosis is endemic in South East Asia and northern Australia, and has a wide range of clinical presentations from mild chronic illness to fulminant infection. Although any organ may be involved, soft tissue and visceral abscesses, pneumonia and septicaemia are particularly common. ${ }^{1}$ Melioidosis is a common cause of morbidity and mortality in the rural, rice farming, communities of northeast Thailand. ${ }^{2}$ Confirmation of the diagnosis by culture requires special media and may take several days. ${ }^{3}$

$P$ pseudomallei is unresponsive to those antibiotics (gentamicin plus a penicillin) commonly used to treat community acquired septicaemia. Therefore, a rapid presumptive diagnosis is essential so that patients may be given appropriate therapy, such as ceftazidime. ${ }^{4}$ Immunofluorescence microscopy to detect whole organism $\mathrm{s}^{5}$ and urinary antigen detection by enzyme linked immunosorbent assay (ELISA) ${ }^{6}$ have been applied to melioidosis but both techniques require expensive equipment, which is not widely available in these endemic areas. Therefore, we have evaluated a simple and rapid latex agglutination test ${ }^{7}$ for the detection of $P$ pseudomallei antigen in urine of patients with melioidosis and compared it with the ELISA.

\section{Methods}

All patients were admitted to Sappasitprasong Hospital, Ubon Ratchatani, between August 1991 and July 1993. Melioidosis was diagnosed definitively by the isolation of $P$ pseudomalle $i$ from clinical specimens as described previously. ${ }^{3}$ Urine specimens were collected on the day of admission from 100 patients with culture positive melioidosis. Of these, 14 had disseminated infection (septicaemia with two or more foci of infection), 40 had septicaemia with one or no focus of infection. Forty five patients had localised $P$ pseudomallei infections including pneumonia or lung abscesses $(\mathrm{n}=$ $23)$, liver or splenic abscesses $(n=7)$, parotid abscesses $(n=5)$, soft tissue and other sites $(n=10)$. The single remaining patient had a localised urinary tract infection $\left(\geq 10^{5}\right.$ colony forming units per $\mathrm{ml}(\mathrm{cfu} / \mathrm{ml})$ ). The mean duration of symptoms before entering the study was 17 days (median 10 days, range two to 100 days).

Urine samples were also obtained from 200 hospitalised patients with a variety of diagnoses other than melioidosis (64 of these had bacteruria $\geq 10^{5} \mathrm{cfu} / \mathrm{ml}$ caused by organisms other than $P$ pseudomallei). This control group of patients came from the same rice farming communities as those with melioidosis.

PREPARATION OF THE LATEX TEST

The latex agglutination test was prepared as described previously. ${ }^{7}$ Briefly, a rabbit polyclonal antibody produced against whole boiled cells of a reference strain of $P$ pseudomallei (NCTC 1688) was used to coat the test latex particles. Control latex particles were coated with normal rabbit serum. Suspensions 
Comparison of latex agglutination and ELISA for detection of P pseudomallei antigen in urine, categorised by site of infection

\begin{tabular}{|c|c|c|c|}
\hline \multirow[b]{2}{*}{ Site of infection } & \multicolumn{2}{|c|}{ Latex agglutination test } & \multirow{2}{*}{$\begin{array}{l}\text { ELISA } \\
\begin{array}{l}(\geq 1 \text { in } 10 \text { dilution } \\
\text { of urine }) \\
n(\%)\end{array}\end{array}$} \\
\hline & $\begin{array}{l}\text { Unconcentrated } \\
\text { urine } \\
n(\%)\end{array}$ & $\begin{array}{l}\text { Concentrated } \\
\text { urine }(\times 100) \\
n(\%)\end{array}$ & \\
\hline Disseminated* & $6 / 14(43)$ & $11 / 14(79)$ & $10 / 10(100)$ \\
\hline Septicaemic & $7 / 40(17 \cdot 5)$ & $23 / 37(62)$ & $26 / 33(79)$ \\
\hline Pulmonary infection & $3 / 23(13)$ & $6 / 22(27)$ & $11 / 21(52)$ \\
\hline \multicolumn{4}{|l|}{ Other localised } \\
\hline infections & $1 / 22(4 \cdot 5)$ & $3 / 19(16)$ & $11 / 17(65)$ \\
\hline Urinary tract infection** & $1 / 1 \quad(100)$ & $1 / 1(100)$ & $1 / 1(100)$ \\
\hline All patients & $18 / 100(18)$ & $44 / 93(47)$ & $58 / 82(71)$ \\
\hline
\end{tabular}

*Disseminated $=$ septicaemia and two or more focal sites of infection

** Urinary tract infection $=P$ pseudomallei $>10^{5} \mathrm{cfu} / \mathrm{ml}$ urine.

were stored at $4{ }^{\circ} \mathrm{C}$ for up to one year and brought to room temperature before use.

\section{LATEX AGGLUTINATION METHOD}

All urine samples were boiled for five minutes to eliminate non-specific reactions, cooled and centrifuged ( $3000 \mathrm{rpm}$ for five minutes) before testing the supernatant fluid. Urine $(40 \mu \mathrm{l})$ was mixed with $20 \mu \mathrm{l}$ latex suspension on a white card. After spreading, the card was rocked gently for three minutes. Agglutination with the test latex but not the control latex indicated a positive result.

Urines samples with sufficient volume $(\geq 5 \mathrm{ml}$ ) were then concentrated 100 -fold in a Minicon B15 concentrator (Amicon Ltd, Stonehouse, UK) before retesting with the latex suspension. This takes approximately 60 to 90 minutes.

\section{ELISA METHOD}

The antigen detection ELISA was performed as described previously. ${ }^{6}$ The ELISA utilises a polyclonal anti- $P$ pseudomallei antibody conjugated to fluoroscein isothiocyanate (FITC) and an anti-FITC antibody amplification system. Urine samples were stored at $-30^{\circ} \mathrm{C}$ and tested in batches. Boiled and centrifuged urine was tested at a range of dilutions.

\section{Results}

A total of 100 unconcentrated test urine samples and 200 control urine samples were analysed. Subsequently, 93 test samples and 40 control samples were concentrated 100 -fold and retested. The results were categorised according to the site of infection (table). The overall sensitivity was $18 \%$ for unconcentrated urine but increased to $47 \%$ for concentrated urine samples. Antigen detection was highest in patients with disseminated or septicaemic infection (34 of $51 ; 67 \%$ ) compared with localised disease ( 10 of $42 ; 22 \%$ ). There were no positive reactions with the 200 control urine samples, including the 40 concentrated samples, giving a specificity of $100 \%$ for the test.

Eighty two test urine samples were available for antigen assay by ELISA. Using the previously recommended cut off dilution of 1 in 10 to ensure high specificity, the overall sensitivity for this group of patients with melioidosis was $71 \%$. Again, the sensitivity was higher in those patients with disseminated infection or septicaemia (36 of $43 ; 84 \%$ ) compared with those with localised infections ( 23 of $39 ; 58 \%$ ).

\section{Discussion}

Severe melioidosis has an extremely high mortality if appropriate treatment is not given promptly. ${ }^{1}$ The current antibiotic of choice, ceftazidime, ${ }^{4}$ is expensive and is not used as first line empirical therapy for community acquired septicaemia. Therefore, rapid tests are required to confirm diagnosis. If suitable specimens such as sputum or pus are available for examination, immunofluorescence microscopy is extremely useful. ${ }^{5}$ Urine is readily available from almost all patients, including children from whom other specimens may be difficult to obtain. The rapid diagnosis of melioidosis by detection of urinary antigen has obvious advantages. An enzyme immunoassay has been developed, ${ }^{6}$ but requires expensive equipment and is most suitable for batch testing.

Latex agglutination tests are used widely for the detection of bacterial antigens in cerebrospinal fluid. They have also been applied to the detection of antigen in urine, with variable results, although sensitivity of the test often improves after concentration of urine. In one study, using urine concentrated 25-fold, Haemophilus influenzae type b antigen was detected in urine in 20 of 23 children with culture positive meningitis or septicaemia. ${ }^{8}$ In a study of pneumococcal pneumonia in Gambian children O'Neill et al' used serotype specific latex tests to detect urinary antigen in $50 \%$ of culture positive cases. The sensitivity of the test increased to $76 \%$ after concentrating the urine 25-fold.

In this study the latex agglutination test, using unconcentrated urine, could detect $P$ pseudomallei antigen only in $18 \%$ of all patients with melioidosis. This increased to $43 \%$ in the group of patients with disseminated disease, presumably reflecting the greater bacterial load and thus higher concentrations of antigen excreted in the urine. Concentrating urine 100fold significantly improved the sensitivity of the test. The increase in sensitivity occurred in patients with localised forms of melioidosis as well as in those with septicaemia. Antigen was still only detected in $47 \%$ patients overall, but increased to over $60 \%$ in patients with septicaemia and nearly $80 \%$ in those with disseminated infection. Therefore, this test will be most useful in those patients with life threatening disease in whom rapid diagnosis and appropriate antibiotic treatment is urgently needed. In the present study the ELISA was considerably better at detecting urinary antigen, although the specificity $(96 \%)$ published previously ${ }^{6}$ is lower than the $100 \%$ specificity for the latex test. The ELISA is also unsuitable for immediate testing of patients on admission to hospital.

This latex agglutination test offers a simple, cheap, rapid method of diagnosing meliodosis. A correct diagnosis may be achieved, within two hours, for two thirds of patients with severe 
melioidosis or septicaemia. It will be particularly useful in laboratories with limited facilities. The cost of concentrating one urine sample 100 -fold is approximately $£ 3$, although it may be possible to increase the sensitivity of the test by other, cheaper methods ${ }^{10}$ making it more widely applicable.

We thank the Director and medical staff of Sappasitprasong Hospital, Ubon Ratchatani, for their support of this study. We are grateful to the nursing staff of the Department of Medicine, and staff of the microbiology laboratory, Sappasitprasong Hospital, for collecting the specimens.

This study was part of the Wellcome-Mahidol University OxThis study ord Tropical Medicine

Trust of Great Britain. Congress of Tropical Medicine and Malaria, Pattaya, Thailand, in December 1992.

1 Leelarasamee A, Bovornkitti S. Melioidosis: review and update. Rev Infect Dis 1989;11:413-25.

2 Chaowagul W, White NJ, Dance DAB, Wattanagoon Y, Naigowit $\mathrm{P}$, Davis TME, et al. Melioidosis: a major cause Naigowit $P$, Davis Thed septicemia in Northeastern Thaiof community-acquired septicemia
land. $\Im$ Infect Dis 1989;159:890-9.
3 Wuthiekanun V, Dance DAB, Wattanagoon Y, Suputtamongkol $Y$, Chaowagul W, White NJ. The use of selective media for the isolation of Pseudomonas of selective media for the isolation of Pseudomonas pseudomall

4 White NJ, Dance DAB, Chaowagul W, Wattanagoon Y, Wuthiekanun V, Pitakwatchara N. Halving of mortality of severe melioidosis by ceftazidime. Lancet 1989;ii:697-701.

5 Walsh AL, Smith MD, Wuthiekanun V, Suputtamongkol Y, Desakorn V, Chaowagul W, et al. Immunofluorescence microscopy for the rapid diagnosis of melioidosis. $f$ Clin Pathol 1994;47:377-9.

6 Desakorn V, Smith MD, Wuthiekanun V, Aucken HM, Suntharasamai P, Rajchanuwong A, et al. Detection of Pseudomonas pseudomallei antigen in urine for the diagnosis of melioidosis. Am ₹ Trop Med Hyg 1995 (in press).

7 Smith MD, Wuthiekanun V, Walsh AL, Pitt TL. A latex agrutination test for the identification of Pseudomonas agglutination test for the identification of
pseudomallei. $\mathcal{F}$ Clin Pathol 1993;46:374-5.

8 Kaldor J, Asznowicz R, Dwyer B. Haemophilus influenzae type b antigenuria in children. F Clin Pathol 1979;32: 538-41.

9 O'Neill KP, Lloyd-Evans N, Campbell H, Forgie IM, Sabally S, Greenwood BM. Latex agglutination test for diagnosing pneumococcal pneumonia in developing countries. $B M \mathcal{F} 1989 ; 298: 1061-4$.

10 Capeding MRZ, Nohynek H, Ruutu P, Leinonen M. Evaluation of a new tube latex agglutination test for detection of type-specific pneumococcal antigens in urine. $\mathfrak{f}$ Clin Microbiol 1991;29:1818-21. 